\title{
CLOTU: An online pipeline for processing and clustering of 454 amplicon reads into OTUs followed by taxonomic annotation
}

\author{
Surendra Kumar ${ }^{1 *}$, Tor Carlsen ${ }^{1}$, Bjørn-Helge Mevik², Pål Enger², Rakel Blaalid ${ }^{1}$, Kamran Shalchian-Tabrizi ${ }^{1}$ and \\ Håvard Kauserud ${ }^{1 *}$
}

\begin{abstract}
Background: The implementation of high throughput sequencing for exploring biodiversity poses high demands on bioinformatics applications for automated data processing. Here we introduce CLOTU, an online and open access pipeline for processing 454 amplicon reads. CLOTU has been constructed to be highly user-friendly and flexible, since different types of analyses are needed for different datasets.

Results: In CLOTU, the user can filter out low quality sequences, trim tags, primers, adaptors, perform clustering of sequence reads, and run BLAST against NCBInr or a customized database in a high performance computing environment. The resulting data may be browsed in a user-friendly manner and easily forwarded to downstream analyses. Although CLOTU is specifically designed for analyzing 454 amplicon reads, other types of DNA sequence data can also be processed. A fungal ITS sequence dataset generated by 454 sequencing of environmental samples is used to demonstrate the utility of CLOTU.

Conclusions: CLOTU is a flexible and easy to use bioinformatics pipeline that includes different options for filtering, trimming, clustering and taxonomic annotation of high throughput sequence reads. Some of these options are not included in comparable pipelines. CLOTU is implemented in a Linux computer cluster and is freely accessible to academic users through the Bioportal web-based bioinformatics service (http://www.bioportal.uio.no).
\end{abstract}

\section{Background}

Microorganisms constitute a large fraction of the biodiversity on earth [1], but the majority of microbial life is still unknown. Improved knowledge about the hidden diversity of microorganisms is vital for a better understanding of evolutionary relationships and ecological processes among microorganisms [2-5]. Sequencing of DNA sampled from the environment has allowed us to venture into this vast diversity of unknown microorganisms. In particular, the introduction of pyrosequencing technologies has revolutionized our ability to explore this hidden diversity [6]. High throughput sequencing of genomic DNA regions such as ITS, $16 \mathrm{~S}$ and $18 \mathrm{~S}$ rDNA enables in-depth analyses of the genetic variation of eukaryotic and prokaryotic groups. These techniques

\footnotetext{
* Correspondence: surendra.kumar@bio.uio.no; havard.kauserud@bio.uio.no 'Microbial Evolution Research Group (MERG), Department of Biology, University of Oslo, P.O. Box 1066 Blindern, N-0316 Oslo, Norway Full list of author information is available at the end of the article
}

have already been exploited to study the microbial community in various environments [6-12].

Analysis of the massive amount of data produced by new sequencing methods requires efficient and flexible bioinformatics applications that both fit the user's needs and the characteristics of the sequence data. There are several existing bioinformatics tools available that include various options for processing and clustering 454 reads, including FASTGROUPII [13], RDP[14], MOTHUR[15], SEQTRIM[16], QIIME[17], SCATA [18], WATERS[19], CANGS[20], PANGEA[21] and PYRONOISE[22]. However, the majority of these programs are directed towards specific genetic markers or include only a few of the necessary analytic steps. Furthermore, some of the analytic steps (i.e. sequence clustering) normally require significant computational power, but many of the published bioinformatics tools are not implemented in a high performance-computing environment and must be installed locally. There is
C Biomed Central 
still a need for a comprehensive, user-friendly and flexible pipeline that transforms raw sequence data (e.g. from 454 GS FLX Titanium pyrosequencing runs or ABI Sanger sequences) into Operational Taxonomic Units (OTUs) and allows the results to be browsed easily.

In this paper we present CLOTU, an online, userfriendly pipeline for processing 454 amplicon reads. CLOTU is open access to academic users and is implemented on the Bioportal bioinformatics web-service (http://www.bioportal.uio.no/). As different users and datasets have different demands, we aimed to make CLOTU as flexible as possible, so analyses can be optimized by adjusting several criteria and parameters. The output of the pipeline shows detailed statistics about the number of sequences passing the different filtering steps, statistics of clusters of sequences (e.g. operational taxonomic units) and BLAST hits.

\section{Methods}

A typical raw 454 read obtained after sequencing with adaptors and tags (named MIDs by Roche) is illustrated in Figure 1. CLOTU includes three basic steps: 1) Filtering and trimming, 2) clustering and 3) database search using BLAST (Figure 2). Each of the three basic steps can be implemented through the web interface independently or collectively, and their respective parameters specified (see additional file 1). A user manual for CLOTU is available on the Bioportal (https://www.bioportal.uio.no/appinfo/show.php?app=clotu).

\section{Input files}

CLOTU requires three input files from the user: 1) one or several sequence files, in FASTA format [23] compressed ZIP file (hereafter referred to as SEQUENCE file), 2) a text file, that specifies sequences used as tags, primers and adaptors (hereafter referred to as TPA file) and 3) a text file, containing the FASTA file names and file identifiers (hereafter referred to as METADATA file) to be added to each sample.

\section{Step1: Filtering and trimming}

CLOTU provides different options for filtering low quality reads. 454 reads in the SEQUENCE file can be removed by the user if: 1) the tag and primer sequences does not match the sequences in the TPA file, 2) sequences have incompatible end tag combinations, 3) one or more ambiguous nucleotides (e.g. Ns) are present $[24,25]$ and 4) sequences are shorter than the userdefined minimum length.

These options can be optimized by the user and implemented either in combination or independently. It is also possible to accept mismatches in tags and primers. A Perl module included in the pipeline implements the Needleman-Wunsch algorithm [26] and considers indels for pairwise alignment of tags and primers before filtering out low quality sequences. The user can also define the threshold for minimal sequence length (default length is 150).

Sequencing by 454 pyrosequencing often results in ambiguous homopolymers. CLOTU provides an option where homopolymers above a certain length can be collapsed to a user-defined length, e.g. all homopolymers of length greater than six can be reduced to length six.

CLOTU allows trimming of tags, primers, and adapters (see additional file 1). One of these options, the 'Trim adaptor' option, removes exact and/or partial adaptor sequences found at the end of the reads. In order to reduce redundancy in the dataset before clustering, CLOTU also includes an option to remove all identical sequences. If this option is selected, CLOTU keeps track of all duplicate sequences and includes them in sequence abundance tallies for each cluster.

The filtering and trimming step produces four or five output files depending on the chosen parameters: 1) summary.txt summarizes the statistics of accepted sequences in tabular format for each basic step of the pipeline, 2) accepted.fas contains all accepted sequences in FASTA format, 3) rejected.fas contains all rejected sequence in FASTA format and 4) stats_log.txt lists the number of sequences in each sequence file compressed

$5^{\prime}$

$3^{\prime}$

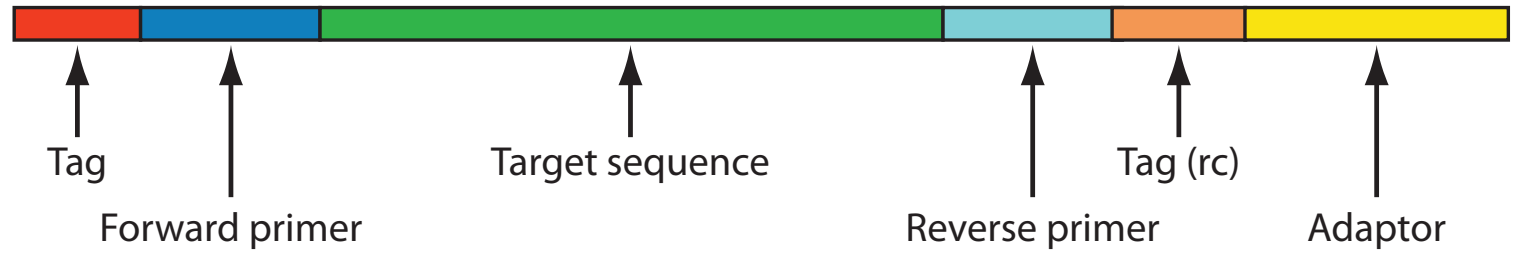

Figure 1 Amplicon sequence structure. Illustration of raw amplicon sequences with tags, primers and adaptors colored in red, blue and yellow respectively. The target sequence amplified by PCR is shown in green color. 


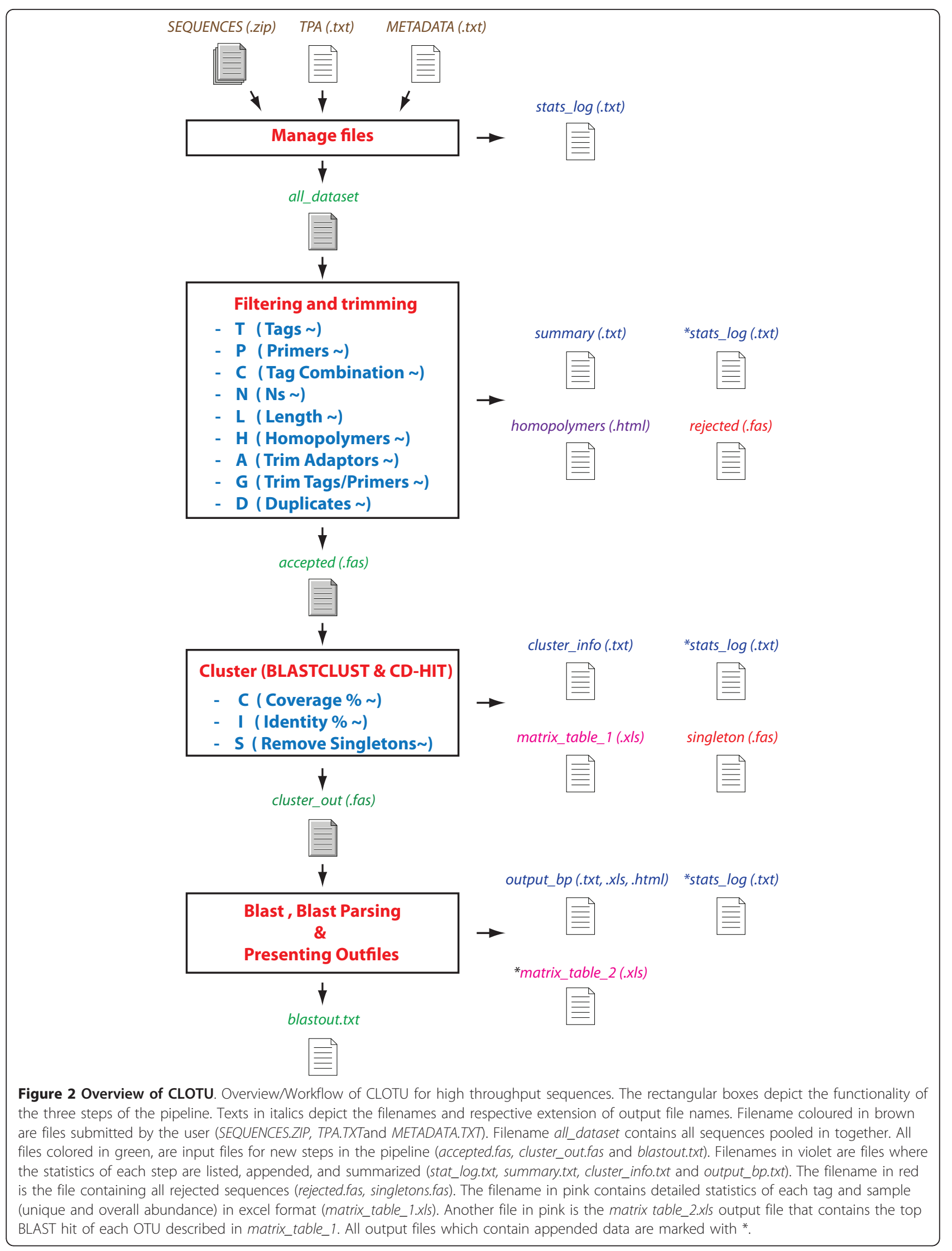


with ZIP. This file includes the parameters selected for the analysis, and detailed statistics regarding the number of accepted and rejected sequences for each of the activated filter and trim parameters. All invalid parameter settings and errors encountered are appended to this file. If the user has activated the 'collapse homopolymer' option, a fifth output file named homopolymers.html is also produced. The homopolymers.html allows visual verification of all sequences with homopolymers (see additional file 2).

\section{Step 2: Clustering of sequence reads}

For clustering of sequence reads, CLOTU uses the single-linkage clustering method as implemented in the BLASTCLUST program. This clusters DNA sequences based on pairwise matches using a BLAST algorithm [27]. The pipeline also provides the option to cluster DNA sequences using the CD-HIT package, an implementation of a greedy incremental clustering algorithm [28]. The user can define the minimum degree of pairwise sequence overlap as well as the sequence similarity threshold for clustering in both algorithms. The output file accepted.fas, containing all accepted sequences, is used as an input file for either clustering program. A typical BLASTCLUST output file consists of a sorted list of clusters of sequences separated by a newline character. The list is sorted first by cluster size and then alphabetically. Sequence identifiers within a cluster are spaceseparated and sorted, first by sequence length and then alphabetically. The longest sequence in each cluster is used as a representative sequence of that cluster. A Perl script creates a ready-to-use FASTA file from the raw BLASTCLUST output. CD-HIT produces ready-to-use FASTA files and the longest sequence from each cluster is considered as the representative sequence. A Perl script numbers the obtained clusters based on their abundance in the complete dataset. The clustering step also provides an option to exclude singletons, frequently used for reducing the impact of PCR and sequencing errors [29].

The clustering step produces five new output files: 1) cluster_out.fas contains the representative sequence of each cluster, 2) cluster_info.txt lists brief statistics about the number of sequences in each cluster and in the whole dataset, 3) matrix_table_1.xls lists the unique and identical (duplicate) sequence count from each tags used in the study, 4) singleton.fas lists all singletons in the FASTA format and 5) SeqInEachCluster.zip which includes separate ready-to-use FASTA formatted files of all sequences in each of the clusters, obtained for further assessment with various multiple sequence analyses or bioinformatics applications. The file stats_log.txt from step 1 is appended with brief statistics on the clusters obtained.

\section{Step 3: Taxonomic annotation of sequences using BLAST}

Taxonomic annotations are done by database searches using BLASTn against either user-defined databases or a downloaded version of the NCBInr database, maintained and updated on the Bioportal server [30]. User-defined databases can be made available for a defined group of users or to all users of the CLOTU pipeline through the Bioportal infrastructure. BLAST searches are done with user-specified settings of E-value threshold, number of score descriptions to report and number of pairwise alignments (see additional file 1). As the NCBInr database contains sequences derived from environmental surveys lacking taxonomical information, the user can choose to remove such hits from the BLAST output files (Perl scripts).

As a rough evaluation of the obtained clusters, CLOTU provides statistics about the degree to which the different clusters have best BLAST hits against the same database sequences. If many clusters have their best hit against the same reference sequences this may indicate that strict clustering parameters have been used, although this might not be universal for other sequences, including ITS.

The BLAST step produces five output files: 1) blastout.txt contains the results from BLAST searches in text format, 2) output_bp.html contains the parsed BLAST search results in color and tabular form, for easy visualization, 3) outfile_bp.txt contains parsed BLAST search results in text format, 4) outfile_bp.xls is a BLAST search result parsed file in Microsoft Excel (tab delimited) format and 5) matrix_table_2.xls is the same as matrix_table_1.xls produced in the previous step with the addition of an extra column for the top BLAST hit (see additional file 3). All significant BLAST search hits reported are summarized and appended in stats_log.txt file. Each of the parsed BLAST output files (outfile_bp $h t m l$, outfile_bp.txt, and outfile_bp.xls) also report all significant hits, along with the top hit that passed the BLAST parsing criteria, as well as brief statistics about the total numbers of hits and number of uncultured sequences reported.

\section{Implementation}

CLOTU is written in Perl v5.8 and PHP 4.3 and implemented on the Bioportal at the University of Oslo. Bioportal is a web-based bioinformatics service and currently the largest high performance-computing environment for bioinformatics in Norway. Bioportal is freely available to academic users at the following URL: http:// www.bioportal.uio.no/. The available computer resources are 593 cores on a TITAN cluster [31] at University of Oslo. In addition, Bioportal has access to all free or idle TITAN cores if needed (approximately 4000 at present). The TITAN cluster has Linux nodes with 16 gigabytes 
of memory and $2 \times$ quadcore CPUs or $2 \times$ dual-core CPUs. The CLOTU and Bioportal tutorials are available at the Bioportal website [32].

\section{Analysed dataset}

A dataset including 12,486 fungal ITS1 rDNA sequences generated by 454 sequencing of eight environmental samples from four plant roots is used here to demonstrate the utility of the CLOTU pipeline. The fungal ITS1 amplicons were obtained through a nested PCR approach using the fungal-specific primer ITS1-F [33] in combination with the primer ITS4 [34] in PCR1 and fusion primers (i.e. including tags and adaptors) based on ITS5 and ITS2 [34] in PCR2. The raw ITS1 sequences consisted of tags, forward primer, target sequence, reverse primer, reverse complement of tags used and adaptor (Figure 1). Tags were used on both ends to be able to control for sequences with incompatible end tag combinations generated during sample pooling for emulsion PCR. Although mainly overseen, such sequences with incompatible tag combinations have been reported as a serious problem in other publications $[35,36]$. The ITS1 dataset has been submitted to GenBank (short read archive) [SRA: SRP006413].

\section{Parameters selected for the analysed dataset}

We did two separate analyses of the ITS1 dataset, each with two different settings, to evaluate and illustrate the different options available in CLOTU. In the first analyses (I) we searched for both the forward (ITS5) and reverse (ITS2) primers within the sequences, in order to filter out those that had not been fully sequenced. We did two separate runs of this analysis: one allowing no errors (mismatches) in the primers, and one allowing for two errors in each primer. In the second analysis (II) we only searched for the forward primer (ITS5), to also retain partially sequenced ITS1 fragments. Again, we did two separate runs in this analysis allowing zero or two mismatches in the forward primer. The four different filtering parameter settings were each used with the two different clustering methods BLASTCLUST and CDHIT. The parameters for BLASTCLUST and CD-HIT were $95 \%, 96 \%, 97 \%, 98 \%$ and $99 \%$ identity and $50 \%$ sequence coverage.

\section{Results and discussion Analyses of the ITS data}

The processing of the fungal ITS1 dataset using different filtering settings is summarized in Table 1 and 2 . About $3.7 \%$ of the sequences were removed, as tags were not detected. Requiring presence of both forward (ITS5) and reverse (ITS2) primers without errors in the sequences (analysis I) resulted in a massive loss of sequences, almost $70 \%$ of the initial sequence number.
Allowing for two errors in the primers reduced this slightly $(67 \%$ loss). When the presence of only the forward primer was allowed (analysis II) only $2 \%$ and $0.2 \%$ of the sequences were filtered out, with no and two base pair primer mismatches, respectively. This indicates that a large proportion of the ITS1 amplicons in this dataset were not sequenced along the entire length. Thus, sequences with incompatible tag combinations were detected only in analysis I (one sequence detected). In analysis I, $2 \%$ of sequences were filtered out due to the presence of IUPAC DNA ambiguity symbols. However, in analysis II, a markedly higher proportion of the sequences contained Ns; about $9 \%$ were filtered out in this step. This indicates that ambiguities are more frequently associated with incompletely sequenced amplicons. Analysis I returned 339 and 571 unique ITS1 sequences while analysis II returned 2,389 and 2,549 sequences (see additional file 4). It is noteworthy that allowing for two mismatches raised the number of retained sequences by only $\sim 1 \%$.

In analysis I the CD-HIT clustering approach yielded almost the same number of clusters as BLASTCLUST irrespective of allowing zero or two bp primer mismatch, except when very stringent parameter settings (98\% and 99\% identity) were used. In analysis II CDHIT yielded more clusters than the BLASTCLUST approach, even when allowing up to $5 \%$ sequence divergence (see additional file 4).

Singletons, i.e. clusters including only one sequence, are to some extent considered a result of PCR and sequencing errors and often omitted from further analysis [29]. The CLOTU pipeline provides a separate FASTA formatted file with all singletons, which enables a separate comparison to the reference sequence database (e.g. NCBInr database) using BLAST. It is noteworthy that most of the top hits were to taxa not covered by the non-singleton clusters. This may reflect poor read quality of the sequences giving rise to random ITS sequences as the best matches [29]. Alternatively, it may indicate the presence of many rare taxa within the samples being studied (see additional file 3: matrix_table_2.xls for the singleton BLAST hit), and that removal of singleton clusters without further assessment in environmental sequencing studies may lead to the loss of valuable information [37]. In CLOTU, the 'remove singleton' option can be deactivated to include the BLAST top hits for even these clusters.

In both analyses I and II, using $98 \%$ and $99 \%$ sequence identity, far more clusters appeared among the sequences when two base pair mismatches were allowed in primers. This may indicate that a higher proportion of low quality sequences have been included when allowing for two base pair errors in primers, resulting in additional clusters. To further evaluate the two clustering methods, 
Table 1 Result summary of ITS data analyses (Filtering step)

\begin{tabular}{|c|c|c|c|c|c|c|}
\hline \multirow[b]{2}{*}{ ANALYSIS I } & \multicolumn{3}{|c|}{ No error allowed } & \multicolumn{3}{|c|}{ Two base pair error allowed } \\
\hline & Considered & Accepted & Rejected & Considered & Accepted & Rejected \\
\hline Tags & 12486 & 12015 & 471 & 12486 & 12015 & 471 \\
\hline Primers (FP+RP) & 12015 & 3285 & 8730 & 12015 & 3656 & 8359 \\
\hline Incompatible tags combination & 3285 & 3284 & 1 & 3656 & 3655 & 1 \\
\hline Ns & 3283 & 3034 & 250 & 3655 & 3399 & 256 \\
\hline Length $(<150)$ & 3034 & 3033 & 1 & 3399 & 3398 & 1 \\
\hline Identical sequences & 3033 & 339 & 2694 & 3398 & 571 & 2827 \\
\hline \multicolumn{7}{|l|}{ ANALYSIS II } \\
\hline Tags & 12486 & 12015 & 471 & 12486 & 12015 & 471 \\
\hline Primers (FP) & 12015 & 11753 & 262 & 12015 & 11988 & 27 \\
\hline Ns & 11753 & 10622 & 1132 & 11988 & 10827 & 1161 \\
\hline Length $(<150)$ & 10622 & 10430 & 192 & 10827 & 10614 & 213 \\
\hline Identical sequences & 10430 & 2389 & 8041 & 10614 & 2549 & 8065 \\
\hline
\end{tabular}

Table providing detailed statistics about the various filtering steps used on the example datasets. In analysis I both forward and reverse primers were searched for in the sequences (with zero or two mismatches in forward and reverse primers). In analysis II, only the forward primer was searched for (with zero or two mismatches in forward primer only). The filtering in analysis I resulted in 339 and 571 unique sequences while analysis II resulted in 2389 and 2549 unique sequences.

BLAST searches were performed on the representative sequences from all clusters obtained using $95 \%$ to $99 \%$ of sequence identity and $40 \%$ to $80 \%$ sequence coverage. The BLAST results showed that stringent clustering parameters (above 50\% coverage and 98\%-99\% identity) had an impact on the number of clusters obtained in BLASTCLUST. CD-HIT was found to be less sensitive in this respect (see Table 2 for details).

In CLOTU, our example dataset with 12,486 sequences took 202 seconds ( $~ 3$ minutes) for analysis I

Table 2 Result summary of ITS data analyses (Clustering step)

\begin{tabular}{|c|c|c|c|c|c|c|c|c|c|c|}
\hline \multirow[b]{2}{*}{ ANALYSIS I ${ }^{1}$} & \multicolumn{5}{|c|}{ No error allowed } & \multicolumn{5}{|c|}{ Two base pair error allowed } \\
\hline & $95 \%$ & $96 \%$ & $97 \%$ & $98 \%$ & $99 \%$ & $95 \%$ & $96 \%$ & $97 \%$ & $98 \%$ & $99 \%$ \\
\hline $\mathrm{TC}_{\mathrm{B}}: \mathrm{TC}_{\mathrm{C}}$ & $12: 12$ & $13: 14$ & $14: 15$ & $17: 18$ & $29: 41$ & $14: 15$ & $15: 17$ & $16: 20$ & $19: 27$ & $41: 68$ \\
\hline $\mathrm{TS}_{\mathrm{B}}: \mathrm{TS}_{\mathrm{C}}$ & $4: 3$ & $4: 4$ & $5: 5$ & $5: 4$ & $13: 14$ & $4: 5$ & $4: 4$ & $4: 6$ & $5: 6$ & $24: 27$ \\
\hline \multicolumn{11}{|c|}{ BLAST ANALYSIS² } \\
\hline $\mathrm{TBH}_{\mathrm{B}}: \mathrm{TBH}_{\mathrm{C}}$ & $5: 6$ & $6: 8$ & $7: 8$ & $8: 9$ & $16: 20$ & $6: 7$ & $7: 9$ & $8: 11$ & $9: 15$ & $24: 39$ \\
\hline TUBH $_{B}:$ TUBH $_{C}$ & $5: 6$ & $6: 8$ & $7: 8$ & $8: 8$ & $11: 11$ & $6: 7$ & $7: 8$ & $8: 10$ & $9: 10$ & $12: 12$ \\
\hline $\mathrm{ES}_{\mathrm{B}}: \mathrm{ES}_{\mathrm{C}}$ & $4: 5$ & $5: 6$ & $6: 6$ & $7: 8$ & $11: 15$ & $4: 5$ & $5: 7$ & $6: 7$ & $7: 11$ & $8: 24$ \\
\hline UES $_{B}: U S_{C}$ & $4: 5$ & $5: 6$ & $6: 6$ & $7: 7$ & $8: 9$ & $4: 5$ & $5: 6$ & $6: 6$ & $7: 8$ & $8: 9$ \\
\hline $\mathrm{OS}_{\mathrm{B}}: \mathrm{OS}_{\mathrm{C}}$ & $1: 1$ & $1: 2$ & $1: 2$ & $1: 1$ & $8: 5$ & $2: 2$ & $2: 2$ & $2: 4$ & $2: 4$ & $16: 15$ \\
\hline UOS $_{B}: U_{C}$ & $1: 1$ & $1: 2$ & $1: 2$ & $1: 1$ & $5: 4$ & $2: 2$ & $2: 2$ & $2: 4$ & $2: 3$ & $6: 5$ \\
\hline \multicolumn{11}{|l|}{ ANALYSIS $\|^{2}$} \\
\hline $\mathrm{TC}_{\mathrm{B}}: \mathrm{TC}_{\mathrm{C}}$ & $40: 70$ & $42: 86$ & $45: 102$ & $58: 177$ & 133:385 & $42: 74$ & $44: 86$ & $47: 106$ & $61: 188$ & $140: 408$ \\
\hline $\mathrm{TS}_{\mathrm{B}}: \mathrm{TS}_{\mathrm{C}}$ & $13: 22$ & $13: 26$ & $14: 31$ & $26: 67$ & $98: 198$ & $14: 25$ & $14: 30$ & $15: 31$ & $28: 68$ & $104: 208$ \\
\hline \multicolumn{11}{|c|}{ BLAST ANALYSIS ${ }^{2}$} \\
\hline $\mathrm{TBH}_{\mathrm{B}}: \mathrm{TBH}_{\mathrm{C}}$ & $25: 52$ & $27: 59$ & $29: 75$ & $36: 133$ & $88: 304$ & $26: 55$ & $28: 63$ & $30: 78$ & $37: 141$ & $92: 322$ \\
\hline TUBH $_{B}:$ TUBH $_{C}$ & $25: 32$ & $27: 35$ & $29: 36$ & $32: 40$ & $41: 45$ & $26: 34$ & $28: 37$ & $30: 37$ & $33: 42$ & $42: 46$ \\
\hline $\mathrm{ES}_{\mathrm{B}}: \mathrm{ES}_{\mathrm{C}}$ & $16: 41$ & $18: 41$ & $20: 52$ & $21: 81$ & $22: 148$ & $17: 37$ & $19: 43$ & $21: 55$ & $22: 91$ & $23: 155$ \\
\hline UES $_{B}: U S_{C}$ & $16: 24$ & $18: 24$ & $20: 26$ & $21: 27$ & $22: 32$ & $17: 23$ & $19: 25$ & $21: 27$ & $22: 30$ & $23: 34$ \\
\hline$O S_{B}: O S_{C}$ & $9: 18$ & $9: 18$ & $9: 23$ & $15: 52$ & $66: 156$ & $9: 18$ & $9: 20$ & $15: 23$ & $69: 50$ & 69:167 \\
\hline $\operatorname{UOS}_{B}: U_{C}$ & $9: 15$ & $9: 15$ & $9: 15$ & $15: 22$ & 29:30 & $9: 15$ & $9: 16$ & $15: 15$ & $29: 23$ & 29:30 \\
\hline
\end{tabular}

Table providing statistics about the clustering steps run on the example dataset. Both BLASTCLUST and CD-HIT were used for clustering the unique sequences (with zero or two mismatches in the forward primer) at 95\%, 96\%, 97\%, 98\%, and 99\% sequence identity and 50\% sequence coverage. The table also summarizes the statistics of BLAST-based evaluation method of clusters.

Subscript B and C means results from BLASTCLUST and CD-HIT respectively.

${ }^{1}$ TC (Total Cluster) is the number of clusters obtained at sequence identity $95 \%, 96 \%, 97 \%, 98 \%$, and $99 \%$ irrespective of number of sequences in each cluster, TS (Total singletons) is the number of cluster with single sequence.

${ }^{2}$ TBH (Total BLAST Hit) is the number of OTUs obtained; TUBH (Total Unique BLAST Hit) is the number of unique BLAST hits obtained from the TBH, ES (Excluding Singletons) is the number of BLAST hits excluding clusters with single sequence (singletons) only; UES is the unique number of BLAST hits obtained from ES; OS (Only Singletons) is the number of BLAST hits obtained from singletons; UOS is the unique number of BLAST hits obtained from OS. 
when the CD-HIT clustering program was selected, and 590 seconds ( 10 minutes) when BLASTCLUST was used. The total time for calculation with either CD-HIT or BLASTCLUST was below 20 seconds without BLAST searches.

\section{CLOTU compared to other bioinformatics tools}

CLOTU is one of a few web-based bioinformatics pipelines that can process raw 454 reads and return taxonomically annotated Operational Taxonomic Units (OTUs) ready for further downstream analyses. CLOTU includes some overlapping functionalities with several recently published pipelines such as the QIIME[17], PANGEA [21], SCATA[18], CANGS[20] and WATERS[19] but is different at some important points (see Table 3). CLOTU is a web-based service platform running on a high performance computing environment, while QIIME, PANGEA, CANGS and WATERS must be installed locally, making subsequent analysis of extensive datasets time consuming.

Compared to other pipelines, CLOTU provides a broad range of filtering options, with many unique functionalities, like filtering based on the presence of one or both primers and sequences with non-congruent tags. Although mainly ignored, it has been shown that sequences with incompatible tag combination can be prevalent in some datasets $[35,36]$. CLOTU also allows the inclusion of a certain number of mismatches in primers as well as tags. The trimming options provided in CLOTU include trimming of only tags or both tags and primers. Furthermore, CLOTU can detect partial adaptors at the end of the sequence when the amplicons are not sequenced completely.

In 454 sequencing, most sequencing errors arise from homopolymer stretches. CLOTU provides the option to collapse homopolymers with user specified settings. As far as we know, among the mentioned pipelines only CLOTU includes this functionality.

CLOTU provides two different clustering methods. BLAST searches with representative sequences from each cluster showed that the two clustering approaches mostly identified the same hits, with a few unique hits for some of clusters obtained using CD-HIT.

PANGEA performs taxonomic annotation of reads and splits the dataset into classified and unclassified reads based on taxonomic affiliation before clustering. We would argue that such a procedure, relying on e.g. GenBank matches, is problematic and may influence the clustering. It seems a better option to cluster sequences prior to taxonomic annotation. CANGS and SEQTRIM do not provide clustering options. In the RDP pipeline, alignment is required before clustering, something that is highly problematic when working with more variable sequences than $16 \mathrm{~S}$.

One of the other useful features of CLOTU is that BLAST is integrated in the pipeline, making it unnecessary for the users to download databases for BLAST searches. In other pipelines such as QIIME, PANGEA, CANGS and PANGEA, the user needs to set up the database and BLAST program on their local computer for assigning taxonomic affiliation to the 454 reads.

Table 3 CLOTU feature comparison with other pipelines

\begin{tabular}{|c|c|c|c|c|c|c|}
\hline PIPELINE PARAMETERS & CLOTU & QIIME & PANGEA & SCATA & CANGS & WATERS \\
\hline 1. Screening and filtering sequences with tags & Yes & Yes & Yes & Yes & Yes & Yes \\
\hline 2. Screening and filtering sequences with primer pair & Yes & Yes & No & ${ }^{1} \mathrm{FP}$ & Yes & ${ }^{1} \mathrm{FP}$ \\
\hline $\begin{array}{l}\text { 3. Screening and filtering sequences with incompatible tags } \\
\text { combination }\end{array}$ & Yes & No & No & No & No & No \\
\hline 4. Screening and filtering sequences with ambiguity & Yes & Yes & Yes & Yes & Yes & ${ }^{2} \mathrm{ND}$ \\
\hline 5. Screening and filtering sequences with length criteria & Yes & Yes & Yes & Yes & Yes & Yes \\
\hline 6. Subsequent trimming tags, primers, and adaptors & Yes & ${ }^{3} \mathrm{P}$ & No & ${ }^{3} \mathrm{P}$ & ${ }^{3} \mathrm{p}$ & No \\
\hline 7. Screening and collapsing homopolymers & Yes & No & No & No & No & No \\
\hline 8. Screening and removing exact identical sequences & Yes & No & No & No & Yes & No \\
\hline 9. Clustering programs ${ }^{4}$ & $B / C$ & $\mathrm{C} / \mathrm{M}$ & $\mathrm{MB} / \mathrm{C}$ & $B L$ & M & $\mathrm{O}$ \\
\hline 10. Removing singletons & Yes & No & No & No & Yes & No \\
\hline 11. BLAST with NCBInr & Yes & Yes & Yes & ${ }^{2} \mathrm{ND}$ & Yes & Yes \\
\hline 12. Filtering uncultured hits from BLAST result files & Yes & No & No & ${ }^{2} \mathrm{ND}$ & No & No \\
\hline 13. Top hit statistics of BLAST results for each OTUs & Yes & No & No & ${ }^{2} \mathrm{ND}$ & No & No \\
\hline 14. Web-service & Yes & No & No & Yes & No & No \\
\hline
\end{tabular}

Table providing comparable overlapping features with other pipelines QIIME, PANGEA, SCATA, CANGS and WATERS.

${ }^{1} \mathrm{FP}=$ Forward primer

${ }^{2} \mathrm{ND}=$ Not documented

${ }^{3} \mathrm{P}=$ Trimming of either tags or primer or adaptor but not all.

${ }^{4}$ Clustering program; $\mathrm{B}=$ BLASTCLUST, $\mathrm{C}=\mathrm{CD}-\mathrm{HIT}, \mathrm{M}=$ MOTHER, MB $=$ MEGABLAST, BL $=$ BLAST and $\mathrm{O}=$ OTUHUNTER 
In contrast to other pipelines, CLOTU provides several output files at every analytical step, allowing the user to explore their data more deeply in addition to obtaining high quality sequence files. CLOTU is available on Bioportal, where output files can be used in several other bioinformatics applications already installed, maintained and routinely updated (see list of applications at http://www.bioportal.uio.no/appinfo/).

\section{Conclusions}

CLOTU has been constructed to be highly flexible so that users can choose different settings for different types of datasets. The user can choose at what stringency level to operate, i.e. whether only high quality long reads will be accepted for further analyses. We recognize that the current research field is developing extremely fast and that new requirements and options must be included in future versions of CLOTU, including novel tools for quality assessment of sequences [22].

\section{Availability and requirements}

Project name: CLOTU version 1.1

Project home page: http://www.bioportal.uio.no

Operating system(s): Platform independent

Programming language: SQL, Perl, Python and PHP

Other requirements: None

License: GNU - GPL

Any restrictions to use by non-academics: Bioportal accepts academic email address only. Test dataset for CLOTU is available at http://www.bioportal.uio.no/onlinemat/online_material.php.

\section{Additional material}

Additional file 1: CLOTU web-interface on the Bioportal. The user can specify input files (i.e. SEQUENCES.ZIP, TPA.TXT and METADATA.TXT). The sequence file must be in the FASTA format and compressed with ZIP. The user can then select different options provided in each step of the CLOTU.

Additional file 2: Output file of CLOTU showing homopolymers as defined by the user (e.g. 8) in red and lower case

Additional file 3: Output file of CLOTU showing the BLAST hits for singletons.

Additional file 4: Result files for analysis I and II.

\section{Acknowledgements}

We acknowledge Tom Kristensen, Anke Stüken, Thomas Haverkamp, Marie Davey, Russell Orr and two anonymous reviewers for valuable comments on the manuscript. Jon Bråte and Dan Kristofer Ree for testing CLOTU. Funding for this work was provided by the University of Oslo, Norway.

\section{Author details}

'Microbial Evolution Research Group (MERG), Department of Biology, University of Oslo, P.O. Box 1066 Blindern, N-0316 Oslo, Norway. ${ }^{2}$ Centre of Information Technology, University of Oslo, Norway.

\section{Authors' contributions}

SK carried out all programming, analysis, and implementation on Bioportal, drafted and wrote the manuscript. HK, TC and KST planned the study, supervised and helped to draft and write the manuscript. RB tested the program on different datasets. BHM and PE contributed with programming and implementation of CLOTU on Bioportal. All authors read and approved the final manuscript.

Received: 28 September 2010 Accepted: 20 May 2011

Published: 20 May 2011

\section{References}

1. Atlas RM, Bartha R: Microbial Ecology. Fundamentals and Applications. Redwood: Benjamin/Cummings; 1993.

2. Whitman WB, Coleman DC, Wiebe WJ: Prokaryotes: the unseen majority. Proc Natl Acad Sci USA 1998, 95(12):6578-6583.

3. Torsvik $V$, Ovreas L: Microbial diversity and function in soil: from genes to ecosystems. Curr Opin Microbiol 2002, 5(3):240-245.

4. Tyson GW, Chapman J, Hugenholtz P, Allen EE, Ram RJ, Richardson PM, Solovyev W, Rubin EM, Rokhsar DS, Banfield JF: Community structure and metabolism through reconstruction of microbial genomes from the environment. Nature 2004, 428(6978):37-43.

5. Gans J, Wolinsky M, Dunbar J: Computational improvements reveal great bacterial diversity and high metal toxicity in soil. Science 2005, 309(5739):1387-1390.

6. Margulies M, Egholm M, Altman WE, Attiya S, Bader JS, Bemben LA, Berka J, Braverman MS, Chen YJ, Chen Z, et al: Genome sequencing in microfabricated high-density picolitre reactors. Nature 2005, 437(7057):376-380.

7. Sogin ML, Morrison HG, Huber JA, Mark Welch D, Huse SM, Neal PR, Arrieta JM, Herndl GJ: Microbial diversity in the deep sea and the underexplored "rare biosphere". Proc Natl Acad Sci USA 2006, 103(32):12115-12120

8. Edwards RA, Rodriguez-Brito B, Wegley L, Haynes M, Breitbart M, Peterson DM, Saar MO, Alexander S, Alexander EC Jr, Rohwer F: Using pyrosequencing to shed light on deep mine microbial ecology. BMC Genomics 2006, 7:57.

9. Roesch LF, Fulthorpe RR, Riva A, Casella G, Hadwin AK, Kent AD, Daroub SH Camargo FA, Farmerie WG, Triplett EW: Pyrosequencing enumerates and contrasts soil microbial diversity. ISME J 2007, 1(4):283-290.

10. Hamady M, Walker JJ, Harris JK, Gold NJ, Knight R: Error-correcting barcoded primers for pyrosequencing hundreds of samples in multiplex. Nat Methods 2008, 5(3):235-237.

11. Bråte J, Logares R, Berney C, Ree DK, Klaveness D, Jakobsen KS, ShalchianTabrizi K: Freshwater Perkinsea and marine-freshwater colonizations revealed by pyrosequencing and phylogeny of environmental rDNA. ISME J 2010

12. Lopez-Garcia P, Moreira D: Tracking microbial biodiversity through molecular and genomic ecology. Res Microbiol 2008, 159(1):67-73.

13. Yu Y, Breitbart M, McNairnie P, Rohwer F: FastGroupll: a web-based bioinformatics platform for analyses of large $16 \mathrm{~S}$ rDNA libraries. BMC Bioinformatics 2006, 7:57.

14. Cole JR, Wang Q, Cardenas E, Fish J, Chai B, Farris RJ, Kulam-SyedMohideen AS, McGarrell DM, Marsh T, Garrity GM, et al: The Ribosomal Database Project: improved alignments and new tools for rRNA analysis. Nucleic Acids Res 2009, 37(Database):D141-145.

15. Schloss PD, Handelsman J: Introducing DOTUR, a computer program for defining operational taxonomic units and estimating species richness. Appl Environ Microbiol 2005, 71(3):1501-1506.

16. Falgueras J, Lara AJ, Fernandez-Pozo N, Canton FR, Perez-Trabado G, Claros MG: SeqTrim: a high-throughput pipeline for pre-processing any type of sequence read. BMC Bioinformatics 2010, 11:38.

17. Caporaso JG, Kuczynski J, Stombaugh J, Bittinger K, Bushman FD, Costello EK, Fierer N, Pena AG, Goodrich JK, Gordon JI, et al: QIIME allows analysis of high-throughput community sequencing data. Nat Methods 2010, 7(5):335-336.

18. SCATA - Sequence Clustering and Analysis of Tagged Amplicons. [http:// scata.mykopat.slu.se/].

19. Hartman AL, Riddle S, McPhillips T, Ludascher B, Eisen JA: Introducing W.A. T.E.R.S.: a workflow for the alignment, taxonomy, and ecology of ribosomal sequences. BMC Bioinformatics 2010, 11:317. 
20. Pandey RV, Nolte V, Schlotterer C: CANGS: a user-friendly utility for processing and analyzing $454 \mathrm{GS}-\mathrm{FLX}$ data in biodiversity studies. BMC Res Notes 2010, 3:3.

21. Giongo A, Crabb DB, Davis-Richardson AG, Chauliac D, Mobberley JM, Gano KA, Mukherjee N, Casella G, Roesch LF, Walts B, et al: PANGEA: pipeline for analysis of next generation amplicons. ISME J 2010.

22. Quince C, Lanzen A, Curtis TP, Davenport RJ, Hall N, Head IM, Read LF, Sloan WT: Accurate determination of microbial diversity from 454 pyrosequencing data. Nat Methods 2009, 6(9):639-641.

23. Pearson WR, Lipman DJ: Improved tools for biological sequence comparison. Proc Natl Acad Sci USA 1988, 85(8):2444-2448.

24. Huse SM, Huber JA, Morrison HG, Sogin ML, Welch DM: Accuracy and quality of massively parallel DNA pyrosequencing. Genome Biol 2007, 8(7):R143.

25. Tedersoo L, Nilsson RH, Abarenkov K, Jairus T, Sadam A, Saar I, Bahram M, Bechem E, Chuyong G, Koljalg U: 454 Pyrosequencing and Sanger sequencing of tropical mycorrhizal fungi provide similar results but reveal substantial methodological biases. New Phytol 2010, 188(1):291-301.

26. Needleman SB, Wunsch CD: A general method applicable to the search for similarities in the amino acid sequence of two proteins. $J \mathrm{Mol}$ Biol 1970, 48(3):443-453.

27. Altschul SF, Madden TL, Schaffer AA, Zhang J, Zhang Z, Miller W, Lipman DJ: Gapped BLAST and PSI-BLAST: a new generation of protein database search programs. Nucleic Acids Res 1997, 25(17):3389-3402.

28. Huang Y, Niu B, Gao Y, Fu L, Li W: CD-HIT Suite: a web server for clustering and comparing biological sequences. Bioinformatics 2010, 26(5):680-682.

29. Dickie IA: Insidious effects of sequencing errors on perceived diversity in molecular surveys. New Phytol 2010, 188(4):916-918.

30. Benson DA, Karsch-Mizrachi I, Lipman DJ, Ostell J, Wheeler DL: GenBank. Nucleic Acids Res 2008, 36(Database):D25-30.

31. Titan. [http://hpc.uio.no/]

32. Kumar $S$, Skjaeveland A, Orr RJ, Enger P, Ruden T, Mevik BH, Burki F, Botnen A, Shalchian-Tabrizi K: AIR: A batch-oriented web program package for construction of supermatrices ready for phylogenomic analyses. BMC Bioinformatics 2009, 10:357.

33. Gardes M, Bruns TD: ITS primers with enhanced specificity for basidiomycetes-application to the identification of mycorrhizae and rusts. Mol Ecol 1993, 2(2):113-118.

34. White T, Bruns T, Lee S, Taylor J: Amplification and direct sequencing of fungal ribosomal RNA genes for phylogenetics. In PCR-protocols a guide to methods and applications. Edited by: Innis MA, Gelfand DH, Sninski JJ, White TJ. San Diego: Acedemic press; 1990:315-322.

35. Binladen J, Gilbert MT, Bollback JP, Panitz F, Bendixen C, Nielsen R, Willerslev E: The use of coded PCR primers enables high-throughput sequencing of multiple homolog amplification products by 454 parallel sequencing. PLoS One 2007, 2(2):e197.

36. van Orsouw NJ, Hogers RC, Janssen A, Yalcin F, Snoeijers S, Verstege E, Schneiders $\mathrm{H}$, van der Poel $\mathrm{H}$, van Oeveren J, Verstegen $\mathrm{H}$, et al: Complexity reduction of polymorphic sequences (CRoPS): a novel approach for large-scale polymorphism discovery in complex genomes. PLoS One 2007, 2(11):e1172.

37. Huse SM, Welch DM, Morrison HG, Sogin ML: Ironing out the wrinkles in the rare biosphere through improved OTU clustering. Environ Microbiol 2010.

doi:10.1186/1471-2105-12-182

Cite this article as: Kumar et al:: CLOTU: An online pipeline for processing and clustering of 454 amplicon reads into OTUs followed by taxonomic annotation. BMC Bioinformatics 2011 12:182.

\section{Submit your next manuscript to BioMed Central and take full advantage of:}

- Convenient online submission

- Thorough peer review

- No space constraints or color figure charges

- Immediate publication on acceptance

- Inclusion in PubMed, CAS, Scopus and Google Scholar

- Research which is freely available for redistribution

Submit your manuscript at www.biomedcentral.com/submit
Biomed Central 